\title{
Metaproteomics of saliva identifies human protein markers specific for individuals with periodontitis and dental caries compared to orally healthy controls
}

\author{
Daniel Belstrøm Corresp., ${ }^{1}$ ， Rosa R. Jersie-Christensen ${ }^{2}$ ， David Lyon ${ }^{3}$ ， Christian Damgaard ${ }^{1}$ ， Lars J. Jensen ${ }^{3}$, \\ Palle Holmstrup ${ }^{1}$, Jesper V. Olsen ${ }^{2}$ \\ ${ }^{1}$ Section of Periodontology and Microbiology, Department of Odontology, Faculty of Health and Medical Sciences, University of Copenhagen, Copenhagen, \\ Denmark \\ 2 Proteomics Program, Novo Nordisk Foundation Center for Protein Research, Faculty of Health and Medical Sciences, University of Copenhagen, \\ Copenhagen, Denmark \\ 3 Disease Systems Biology Program, Novo Nordisk Foundation Center for Protein Research, Faculty of Health and Medical Sciences, University of \\ Copenhagen, Copenhagen, Denmark \\ Corresponding Author: Daniel Belstrøm \\ Email address: dbel@sund.ku.dk
}

Abstract Background: The composition of the salivary microbiota has been reported to differentiate between patients with periodontitis, dental caries and orally healthy individuals. To identify characteristics of diseased and healthy saliva we thus wanted to compare saliva metaproteomes from patients with periodontitis and dental caries to healthy individuals. Methods: Stimulated saliva samples were collected from 10 patients with periodontitis, 10 patients with dental caries and 10 orally healthy individuals. The proteins in the saliva samples were subjected to denaturing buffer and digested enzymatically with LysC and trypsin. The resulting peptide mixtures were cleaned up by solid-phase extraction and separated online with $2 \mathrm{~h}$ gradients by nano-scale $\mathrm{C}_{18}$ reversedphase chromatography connected to a mass spectrometer through an electrospray source. The eluting peptides were analyzed on a tandem mass spectrometer operated in datadependent acquisition mode. Results: We identified a total of 35664 unique peptides from 4161 different proteins, of which 1946 and 2090 were of bacterial and human origin, respectively. The human protein profiles displayed significant overexpression of the complement system and inflammatory markers in periodontitis and dental caries compared to healthy controls. Bacterial proteome profiles and functional annotation were very similar in health and disease. Conclusions: Overexpression of proteins related to the complement system and inflammation seems to correlate with oral disease status. Similar bacterial proteomes in healthy and diseased individuals suggests that the salivary microbiota predominantly thrives in a planktonic state expressing no disease-associated characteristics of metabolic activity. 
1 Metaproteomics of saliva identifies human protein markers specific for individuals with

2 periodontitis and dental caries compared to orally healthy controls

4 Daniel Belstrøm ${ }^{1 *}$, Rosa R. Jersie-Christensen ${ }^{2 *}$, David Lyon ${ }^{3}$, Christian Damgaard ${ }^{1}$, Lars J.

5 Jensen $^{3}$, Palle Holmstrup ${ }^{1}$, Jesper V. Olsen²

6

$7 \quad *$ : these authors contributed equally to this manuscript.

8 1. Section of Periodontology and Microbiology, Department of Odontology, Faculty of Health

9 and Medical Sciences, University of Copenhagen, Denmark.

10 2. Proteomics Program, Novo Nordisk Foundation Center for Protein Research, Faculty of

11 Health and Medical Sciences, University of Copenhagen, Blegdamsvej 3B, DK-2200

12 Copenhagen, Denmark.

13 3. Disease Systems Biology Program, Novo Nordisk Foundation Center for Protein Research,

14 Faculty of Health and Medical Sciences, University of Copenhagen, Blegdamsvej 3B, DK-2200

15 Copenhagen, Denmark.

16 Corresponding author

17 Daniel Belstrøm ${ }^{1}$

18 E-mail: dbel@sund.ku.dk 


\section{Abstract}

25 Background: The composition of the salivary microbiota has been reported to differentiate 26 between patients with periodontitis, dental caries and orally healthy individuals. To identify characteristics of diseased and healthy saliva we thus wanted to compare saliva metaproteomes from patients with periodontitis and dental caries to healthy individuals.

Methods: Stimulated saliva samples were collected from 10 patients with periodontitis, 10 patients with dental caries and 10 orally healthy individuals. The proteins in the saliva samples were subjected to denaturing buffer and digested enzymatically with LysC and trypsin. The resulting peptide mixtures were cleaned up by solid-phase extraction and separated online with $2 \mathrm{~h}$ gradients by nano-scale $\mathrm{C}_{18}$ reversed-phase chromatography connected to a mass spectrometer through an electrospray source. The eluting peptides were analyzed on a tandem mass spectrometer operated in data-dependent acquisition mode.

Results: We identified a total of 35664 unique peptides from 4161 different proteins, of which 1946 and 2090 were of bacterial and human origin, respectively. The human protein profiles displayed significant overexpression of the complement system and inflammatory markers in periodontitis and dental caries compared to healthy controls. Bacterial proteome profiles and functional annotation were very similar in health and disease.

Conclusions: Overexpression of proteins related to the complement system and inflammation seems to correlate with oral disease status. Similar bacterial proteomes in healthy and diseased individuals suggests that the salivary microbiota predominantly thrives in a planktonic state expressing no disease-associated characteristics of metabolic activity. 
46

47 Saliva is a biological fluid critically involved in maintenance of oral homeostasis [1], as

48 qualitative and quantitative changes of saliva associates with increased frequency and severity of

49

50

51

52

53

54

55

56

57

58

59

60 diseases in the oral cavity [2,3]. Furthermore, saliva is easily and non-invasively collected [4], making it interesting to screen for biomarkers associated with oral and general health and disease status $[5,6]$.

In the last decade salivary biomarkers of periodontitis and dental caries have been intensively investigated $[7,8]$. These include salivary bacterial profiles that differentiate in patients with periodontitis [9-11], dental caries [12-14] and orally healthy individuals. Furthermore, increased salivary levels of inflammatory protein biomarkers such as interleukin-1 $\beta$ (IL-1 $\beta$ ), IL-6 and matrix metalloproteinase-8 (MMP-8) have been described to be associated with periodontal disease status [15-18]. Recently, the salivary transcriptome has been assessed [19], and some transcriptomic characteristics of saliva have been reported in patients with dental caries [20]. Collectively, these reports conclude that biomarkers of different biological origin may be adequately assessed in saliva samples and support the concept that the biological composition of saliva reflects individual oral health status.

Mass spectrometry-based proteomics enables characterization of the protein content in any sample, including proteins of human and bacterial origin. It thus provides the possibility for simultaneous characterization of bacterial and host specific differences of saliva associated with oral health and disease. Only three studies have so far attempted to perform metaproteomic analysis of saliva in oral health [21-23]. To the best of our knowledge, no study has so far compared metaproteomic profiles of saliva from patients with periodontitis and dental caries to orally healthy individuals.

The aim of the present study was to characterize the salivary metaproteome in 30 saliva samples, and compare human and bacterial proteome profiles between patients with periodontitis, dental caries and orally healthy individuals. The hypothesis was that both bacterial and human subsets of salivary metaproteome would differentiate between individuals with different oral health status. 


\section{Study population and sample collection}

The study population, clinical examination and collection of saliva samples have been presented in detail [11]. In brief, saliva production was induced by chewing on a tasteless paraffin gum, and chewing-stimulated saliva samples were collected from 10 patients with periodontitis, 10 patients with dental caries and 10 orally healthy individuals following a standardized protocol [24]. Immediately after collection saliva samples were divided into four aliquots and stored at $-80^{\circ} \mathrm{C}$ for further analysis. One aliquot has previously been analyzed by next-generation sequencing (the Human Oral Microbe Identification using Next Generation Sequencing, HOMINGS) [11]. All participants signed an informed consent prior to participation, and the study was approved by the regional ethical committee (H-15000856-53175) and reported to the Danish Data Authorization (2015-54-0970).

\section{Sample preparation}

The saliva proteome samples were prepared as described in [25] with a few modifications. Briefly, $1 \mathrm{ml}$ of saliva was mixed with $1.5 \mathrm{ml}$ lysis buffer (9M Guanidine hydrochloride, $10 \mathrm{mM}$ Chloroacetamide, 5mM tris(2-carboxyethyl)phosphine in 100mM Tris $\mathrm{pH} 8.5$ ) and heated for 10 $\min \left(99^{\circ} \mathrm{C}\right)$ followed by $4 \mathrm{~min}$ of sonication.

Protein concentration was measured with Bradford protein assay and ranged from $1 \mathrm{mg} / \mathrm{ml}$ to 2.5 $\mathrm{mg} / \mathrm{ml}$. All samples were digested with the same amount of Lysyl Endoproteinase (Wako, Osaka, Japan) in a ratio of 1:100 w/w calculated from the highest concentration for $2 \mathrm{~h}$. Samples were diluted to a final volume of $10 \mathrm{ml}$ with $25 \mathrm{mM}$ Tris $\mathrm{pH} 8$ and digested overnight with Trypsin (modified sequencing grade, Sigma) in a 1:100 w/w ratio.

Digestion was quenched by adding $1 \mathrm{ml}$ of $10 \%$ trifluoroacetic acid and centrifuged at $2000 \mathrm{~g}$ for $5 \mathrm{~min}$. The resulting soluble peptides in the supernatant were desalted and concentrated on Waters Sep-Pak reversed-phase $\mathrm{C}_{18}$ cartridges (one per sample) and the tryptic peptide mixtures were eluted with $40 \%$ acetonitrile $(\mathrm{ACN})$ followed by $60 \% \mathrm{ACN}$. Peptide concentrations were determined by NanoDrop (Thermo, Wilmington, DE) measurement. 
103

104

105

106

107

108

109

110

111

112

113

114

115

116

117

118

119

120

121

122

123

124

125

126

127

128

129

130

131

\section{Mass spectrometry analysis}

$1.5 \mu \mathrm{g}$ peptide mixture from each sample was analyzed by online nano-scale liquid chromatography tandem mass spectrometry (LC-MS/MS) in turn. Peptides were separated on an in-house packed $50 \mathrm{~cm}$ capillary column with $1.9 \mu \mathrm{m}$ Reprosil-Pur $\mathrm{C}_{18}$ beads using an EASYnLC 1000 system (Thermo Scientific). The column temperature was maintained at $50^{\circ} \mathrm{C}$ using an integrated column oven (PRSO-V1, Sonation GmbH, Biberach, Germany). Buffer A consisted of $0.1 \%$ Formic Acid, and Buffer B of $80 \%$ ACN, 0.1\% Formic Acid. The flow rate of the gradient was $200 \mathrm{nl} / \mathrm{min}$ and started at 5\% buffer B, going to $25 \%$ buffer B in $110 \mathrm{~min}$, followed by a 25 min step going to $40 \%$ buffer B and continuing to $80 \%$ buffer B in 5 min for a 5 min wash and returning to $5 \%$ in $5 \mathrm{~min}$ and continuing for re-equilibration for $5 \mathrm{~min}$.

The Q Exactive HF instrument (Thermo Scientific, Bremen, Germany) was run in a data dependent acquisition mode using a top 12 Higher-Collisional Dissociation (HCD)-MS/MS method with the following settings. Spray voltage was set to $2 \mathrm{kV}$, S-lens RF level at 50, and heated capillary at $275^{\circ} \mathrm{C}$. Full scan resolutions were set to and 60,000 at $\mathrm{m} / \mathrm{z} 200$ and scan target was $3 \times 10^{6}$ with a maximum fill time of $20 \mathrm{~ms}$. Full-scan MS mass range was set to 3001750 and dynamic exclusion to $20 \mathrm{~s}$. Target value for HCD-MS/MS scans was set at $1 \times 10^{5}$ with a resolution of 30,000 and a maximum fill time of $60 \mathrm{~ms}$. Normalized collision energy was set at 28.

\section{Data analysis}

All 30 raw LC-MS/MS data files were processed together using MaxQuant version 1.5.0.38 [26] with default settings and match between runs. The integrated Andromeda peptide search engine and a reversed database approach applying a 1\% FDR at both peptide and protein level was used. The data was searched in two iterations analogous to a previously described metaproteomics database search strategy [27] First, the search space consisted of the full SwissProt protein database [28] and the Human Oral Microbiome database [29] (both downloaded August 2014). The resulting search output was then used for reduction of the search space after filtering on different parameters. As a quality control measure proteins with less than 2 unique peptides were removed. Furthermore, we required proteins to be detected in at least 5 out of 30 samples. Accession numbers from the Majority protein IDs column in the proteinGroups.txt were used to 
132 retrieve information about Lowest Common Ancestor (LCA) for each protein group entry. To

133 find the Lowest Common Ancestor (LCA) of a protein group, accession numbers with the most 134 peptide-associations were selected, mapped to species and their full taxonomic lineage. The 135 lowest taxonomic rank of the intersection of the latter yielded the LCA. All LCA searches

136 resulting in the parvorder Catarrhini (primates) were set to be human. LCAs at taxonomic rank of 137 species and genera, as well as all of their descendants were used to create a new, reduced search 138 space. The latter was used for the second iteration of MS data identification and quantification 139 and all accession numbers within a protein group were used to perform LCA searches. The above 140 functionality was achieved using Python. Species names from SwissProt and HOMD were 141 mapped to NCBI taxonomic identifiers using UniProt (http://www.uniprot.org/docs/speclist) and 142 NCBI resources (http://www.ncbi.nlm.nih.gov/Taxonomy/TaxIdentifier/tax_identifier.cgi), 143 respectively. Full taxonomic lineages were retrieved from NCBI Taxonomy database dump files 144 (ftp://ftp.ncbi.nlm.nih.gov/pub/taxonomy/). Taxonomic comparison at genus- and species level 145 was performed using Mann-Whitney U test with Benjamini-Hochberg correction for multiple 146 testing.

147 Protein intensities based on summed peptide MS signal intensities were quantile normalized 148 using the limma package version 3.24.15 under R version 3.2.2. Only proteins identified with more than one peptide ("razor+unique") and present in more than five out of the 30 samples were considered for further analysis. The mass spectrometry proteomics data have been deposited to the ProteomeXchange Consortium via the PRIDE [30] partner repository with the dataset 152 identifier PXD004319.

153 154 155 156
For comparative analysis of the human protein profiles, the normalized intensity values were $\log 2$ transformed. For principal component analysis (PCA), missing values were replaced with the constant value 19, representing the lowest protein intensity value measured. Analysis of significance (ANOVA) between groups was performed with the software package Perseus (http://coxdocs.org/doku.php?id=perseus:start). The resulting differentially expressed proteins were clustered using Euclidian distance after scaling the data by subtracting the mean intensity value. All p-values were corrected for multiple comparisons. 
161

162

163

164

165

166

167

168

169

170

171

172

173

174

175

176

177

178

179

180

181

182

\section{Functional annotation of bacterial proteins}

Bacterial proteins from HOMD were searched against Hidden Markov Models (HMMs) of bacterial Nested Orthologous Groups (NOGs) from eggNOG [31] using HMMscan version 3.1 (http://hmmer.org)[32]. For each protein query the resulting hits were restricted by two criteria. E-values had to be equal or lower than 1e-4 and a maximum overlap of 8 amino acids of HMMs was allowed (selecting hits with the lowest e-value). All corresponding NOG-names were used to retrieve Gene Ontology (GO)-terms as well as KEGG pathways from eggNOG.

KEGG pathway enrichment and characterization

To gain insights into differences between the three sample groups, KEGG pathway enrichment was performed using a modified version of AGOtool [33]. Individual samples were grouped to sample categories and the three paired combinations used for the enrichment analysis. All bacterial protein groups with an LCA at rank genus or below were selected. BenjaminiHochberg correction (FDR) of p-values was applied to correct for multiple testing. The FDR was set to $1 \%$. The following additional filter criterion was applied. The fold change had to be equal or higher than 2 or equal or lower than 0.5 .

To get a functional overview of the bacterial proteins, we characterized each individual sample group by counting the number of protein groups associated with each KEGG pathway. For visualization purposes (Fig. S2), we selected the most highly associated terms. Within each group the number of associations was converted to percentages, and the most highly associated terms retained, until a cumulative sum of $90 \%$ was reached. This reduced the number of KEGG terms from 135 to 50 . 


\section{Results}

\section{General findings}

185 Biomass analyses based on summed protein intensity measures demonstrated that approximately $18695 \%$ and $3 \%$ of the total protein biomass was of human and bacterial origin, respectively (Fig.

187 1). Food-related proteins and proteins that could not be assigned to kingdom level comprised the 188 rest of the biomass. We identified a total of 35664 unique peptides from 4161 proteins, of which $97 \%$ of the identified proteins could be assigned as bacterial or human proteins, with almost equal numbers of the two (Fig. 1 and Table 1). The protein biomass and numbers between groups showed no significant differences using ANOVA analysis (Fig. S1).

192

193

194

195

196

197

198

199

200

201

202

203

204

205

206

207

208

209

210

\section{Human protein profiling}

Principal component analysis (Fig. 2A) of the human proteins in saliva showed decent separation of samples from patients with periodontitis and dental caries from orally healthy individuals, based on the most decisive component of the dataset, accounting for $17.9 \%$ of the variation. The most enriched KEGG pathway in component 1 and 2 was 'Complement and coagulation cascades' (Fig. 2B). Component 2 also separated samples from patients with dental caries and periodontitis patients from orally healthy individuals with the component explaining $12.7 \%$ of the variation. Two of the most enriched terms in component 2 in the direction of the orally healthy individuals were KEGG pathway 'Salivary secretion' and GOBP ‘protein glycosylation'.

From a total of 2090 identified proteins of human origin, 60 proteins were significantly differentially expressed when performing multiple sample test (ANOVA, $p<0.05$ ). Hierarchical cluster analysis of the proteins nicely separated the three sample groups, although 3 periodontitis individuals cluster together with the healthy group (Fig. 3). We identified three main protein clusters. Cluster I contains human proteins that are higher expressed in both disease groups compared to controls, and 10 of 20 proteins in this cluster are associated with the GO term 'innate immune response' (protein name in purple). Cluster II consist of 9 proteins that distinguish the individuals with caries from the other groups. In cluster III the protein intensities in the caries group are higher than the mean, for the orally healthy group it is around the mean and for the individuals with periodontitis lower. 
211

212 Of the 1946 proteins of bacterial origin identified, approximately $92 \%$ and $34 \%$ could be

213 assigned to genus and species level, respectively (Table 1). A total of 29 different bacterial

\section{Bacterial protein profiling} genera and 81 species were identified. The five most predominant bacterial genera were

Streptococcus, Prevotella, Veillonella, Rothia and Neisseria collectively representing approx. $70 \%$ of the total bacterial mass. The five most predominant bacterial species identified were Rothia mucilanginosa, Veillonella atypica, Prevotella histicola, Prevotella melaninogenica and Streptococcus salivarius. Abundances of the 20 most predominant bacterial genera and species are displayed in Fig. 4A-B. No statistically significant differences were observed between groups at genus or species level. However, at genus level there is a trend of higher proportion of Veillonella and lower proportion of Haemophilus were associated with dental caries and higher proportions of Fusobacterium, Leptotrichia and Selenomonas and lower proportions of Streptococcus, Rothia and Haemophilus were associated with periodontitis, when compared to orally healthy individuals. The same trend is seen at species level where higher proportion of Veillonella atypica and lower proportion of Haemophilus parainfluenzae were associated with dental caries, and higher proportions of Fusobacterium periodonticum and Leptotrichia wadei and lower proportions of Haemophilus parainfluenzae were associated with periodontitis, when compared to orally healthy individuals. A full list of all bacterial genera and species identified are presented in Table S1.

\section{$K E G G$ pathway enrichment for bacterial proteins}

KEGG pathway enrichment analysis of bacterial proteins resulted in no significant differences with the application of the previously mentioned fold-change and FDR filter criteria. The characterization of functional associations of bacterial proteins is shown in Fig. S2. 


\section{Discussion}

236 The purpose of the present study was to compare metaproteome profiles of saliva from patients

237 with periodontitis or dental caries to that of orally healthy individuals, as we hypothesized that

238 the composition of the salivary metaproteome would associate with oral health status. To the best

239 of our knowledge, this is the first study to characterize both human and bacterial parts of the

240 salivary metaproteome in patients with periodontitis and dental caries.

241 In this study, proteins of bacterial origin constituted $46 \%$ of the proteome diversity, despite only

$2423 \%$ of the total biomass being bacterial. This agrees with the previously reported approx. $1 \%$ of

243 DNA in saliva being of bacterial origin [34].

244 We identify 1946 different bacterial proteins, which is in the same range as the pioneering

245 studies of the salivary metaproteome $[21,22]$ but substantially higher than in dental plaque (983

246 proteins) [35]. From the total 1946 bacterial proteins, 92\% and 34\% could be assigned to genus

247 and species level, respectively (Table 1). The percentage of bacterial proteins identified at genus

248 and species level is considerably higher than what has previously been accomplished in

249 metaproteomic analysis of saliva [21,22]. Streptococcus, Prevotella, Veillonella, Rothia and

250 Neisseria were the most predominant genera identified, constituting approx. $70 \%$ of the biomass

251 across all samples (Fig 4A). This phylogenetic distribution is in line with analysis of the same

252 samples using next-generation sequencing [11], and with previous metaproteomic analysis of

253 saliva in oral health [21-23]. By contrast, an analysis of 17 plaque samples from patients with

254 dental caries and healthy controls by metagenomics, metatranscriptomics and metaproteomics

255 found different bacterial compositions in dental plaque at DNA, mRNA and protein level [35].

256 This may reflect differences between studying the metabolically active dental plaque biofilm and

257 the planktonic, metabolically inactive state of the salivary microbiota, and it is in concordance

258 with the functional annotation analysis performed (Fig. S2). Moreover, the finding of higher

259 proportions of Veillonella in saliva samples from patients with caries and higher proportions of

260 Fusobacterium in samples from periodontitis patients confirms findings from 16S analysis of the

261 same samples [11]. Interestingly, specific oral bacterial species such as Veillonella parvula and

262 Fusobacterium periodonticum have been reported to associate with dental caries and

263 periodontitis, respectively [36,37]. 
264 Furthermore, 2090 different proteins of human origin were identified, which is more than in 265 metaproteome profiling of dental plaque [35] and less than a recent study that identified more 266 than 3700 different human proteins in a mouth swab analysis [23]. The higher number of human 267 protein identifications in mouth swabs is probably due to swabbing the inside of the complete 268 oral cavity including the inside of the cheek. In this study we used stimulated saliva samples, 269 which may have diluted the concentration of proteins within the samples compared to that of 270 unstimulated saliva $[38,39]$. This will of course also affect number of identifications. Based on 271 this finding, unstimulated saliva samples may be preferred for in-depth analysis of the salivary proteome. However, as collection of unstimulated saliva samples is considerably more intricate and time-consuming than collection of stimulated saliva samples, the feasibility of using unstimulated saliva samples for population-based biomarker screening approaches may be limited [11]. In addition, we have recently compared the salivary microbiota in unstimulated and stimulated saliva samples, collected from the same individuals, and reported that comparable microbiotas could be identified using the two types of samples [40]. Consequently, stimulated saliva samples were used in this study.

Data on the human profile of the salivary metaproteome showed differences between oral health and disease, as proteins involved in innate immunity and inflammatory proteins were more abundantly expressed in saliva samples from patients with periodontitis and dental caries than orally healthy individuals (Fig 3). Thus, by use of a contemporary metaproteomics approach we were able to explore that salivary expression of proteins from the innate immune system associates with periodontitis and dental caries. Interestingly, these data are in line with previous reports on periodontitis patients $[41,42]$. Likewise, it has been reported that active components of the complement system in the gingival crevicular fluid associates with both periodontitis $[43,44]$ and gingivitis $[45,46]$. Increased local activation of the complement system in the periodontal tissues increases vascular permeability, vasodilatation and recruitment of inflammatory cells, resulting in excessive release of reactive oxygen species, proteolytic enzymes and interleukins [47-49]. Furthermore, serum levels of complement proteins, has been suggested to express a linear relationship with the degree of periodontal inflammation [50]. Gingivitis is a mild form of gum disease that results in irritation, redness and swelling caused by inflammation of the gums. Thus, the abundant expression of complement proteins and inflammatory mediators in saliva might reflect either a spillover from the gingival crevicular fluid, or alternatively, mirror 
295 increased serum levels of these proteins. Notably, while the complement system has been

296 acknowledged to have a profound role in the pathogenesis of periodontitis [51], the complement

297 system seems to have limited impact on development of dental caries. The expression of

298 complement proteins and other inflammatory proteins in saliva from patients with dental caries is

299 most likely associated with gingivitis in the periodontal tissues adjacent to approximal and

300 gingival caries lesions, and presumably not directly associated with presence of dental caries as

301 such.

\section{Conclusion}

303 Quantitative proteomics data from the present investigation suggest that the salivary microbiota

304 predominantly thrives in a planktonic state with limited metabolic activity, as comparable

305 microbial compositions of the salivary microbiota were obtained based on different omics

306 analysis. Thus, the bacterial part of the metaproteome seems to be inadequate for biomarker

307 analysis of periodontitis and caries. Conversely, a set of human proteins hold the potential to be

308 used as future biomarkers of oral disease status. However, the cross-sectional study design

309 obviously hampers the possibility to address causality of this observation. Thus, future large-

310 scale longitudinal studies of human saliva proteome changes are warranted to reveal the full

311 potential of quantitative proteomics of saliva as a technique to discover biomarkers of oral health

312 and disease. 
1. Marsh PD, Do T, Beighton D, Devine DA (2016) Influence of saliva on the oral microbiota. Periodontol 2000 70: 80-92. 10.1111/prd.12098 [doi].

319

320

321

322

323

324

325

326

327

328

329

330

331

332

333

334

335

336

337

338

339

340

341

342

343

344

345

346

347

348

349

350

2. Dawes C, Pedersen AM, Villa A, Ekstrom J, Proctor GB, Vissink A, Aframian D, McGowan R, Aliko A, Narayana N, Sia YW, Joshi RK, JENSEN SB, Kerr AR, Wolff A (2015) The functions of human saliva: A review sponsored by the World Workshop on Oral Medicine VI. Arch Oral Biol 60: 863-874. S0003-9969(15)00069-2 [pii];10.1016/j.archoralbio.2015.03.004 [doi].

3. Almstahl A, Wikstrom M (1999) Oral microflora in subjects with reduced salivary secretion. J Dent Res 78: 1410-1416.

4. Giannobile WV, McDevitt JT, Niedbala RS, Malamud D (2011) Translational and clinical applications of salivary diagnostics. Adv Dent Res 23: 375-380. 23/4/375

[pii];10.1177/0022034511420434 [doi].

5. Baum BJ, Yates JR, III, Srivastava S, Wong DT, Melvin JE (2011) Scientific frontiers: emerging technologies for salivary diagnostics. Adv Dent Res 23: 360-368. 23/4/360 [pii];10.1177/0022034511420433 [doi].

6. Zhang Y, Sun J, Lin CC, Abemayor E, Wang MB, Wong DT (2016) The emerging landscape of salivary diagnostics. Periodontol 2000 70: 38-52. 10.1111/prd.12099 [doi].

7. Yoshizawa JM, Schafer CA, Schafer JJ, Farrell JJ, Paster BJ, Wong DT (2013) Salivary biomarkers: toward future clinical and diagnostic utilities. Clin Microbiol Rev 26: 781-791. 26/4/781 [pii];10.1128/CMR.00021-13 [doi].

8. Miller CS, Foley JD, Bailey AL, Campell CL, Humphries RL, Christodoulides N, Floriano PN, Simmons G, Bhagwandin B, Jacobson JW, Redding SW, Ebersole JL, McDevitt JT (2010) Current developments in salivary diagnostics. Biomark Med 4: 171-189.

9. Paju S, Pussinen PJ, Suominen-Taipale L, Hyvonen M, Knuuttila M, Kononen E (2009) Detection of multiple pathogenic species in saliva is associated with periodontal infection in adults. J Clin Microbiol 47: 235-238. JCM.01824-08 [pii];10.1128/JCM.01824-08 [doi].

10. Belstrom D, Fiehn NE, Nielsen CH, Kirkby N, Twetman S, Klepac-Ceraj V, Paster BJ, Holmstrup P (2014) Differences in bacterial saliva profile between periodontitis patients and a control cohort. J Clin Periodontol 41: 104-112. 10.1111/jcpe.12190 [doi].

11. Belstrom D, Paster BJ, Fiehn NE, Bardow A, Holmstrup P (2016) Salivary bacterial fingerprints of established oral disease revealed by the Human Oral Microbe Identification using Next Generation Sequencing (HOMINGS) technique. J Oral Microbiol 8: 30170.30170 [pii].

12. Yang F, Zeng X, Ning K, Liu KL, Lo CC, Wang W, Chen J, Wang D, Huang R, Chang X, Chain PS, Xie G, Ling J, Xu J (2012) Saliva microbiomes distinguish caries-active from healthy human populations. ISME J 6: 1-10. ismej201171 [pii];10.1038/ismej.2011.71 [doi]. 
13. Belstrom D, Fiehn NE, Nielsen CH, Holmstrup P, Kirkby N, Klepac-Ceraj V, Paster BJ, Twetman S (2014) Altered Bacterial Profiles in Saliva from Adults with Caries Lesions: A Case-Cohort Study. Caries Res 48: 368-375. 000357502 [pii];10.1159/000357502 [doi].

14. Belstrom D, Fiehn NE, Nielsen CH, Klepac-Ceraj V, Paster BJ, Twetman S, Holmstrup P (2015) Differentiation of salivary bacterial profiles of subjects with periodontitis and dental caries. J Oral Microbiol 7: 27429. 27429 [pii].

15. Kinney JS, Morelli T, Braun T, Ramseier CA, Herr AE, Sugai JV, Shelburne CE, Rayburn LA, Singh AK, Giannobile WV (2011) Saliva/pathogen biomarker signatures and periodontal disease progression. J Dent Res 90: 752-758. 0022034511399908 [pii];10.1177/0022034511399908 [doi].

16. Ebersole JL, Schuster JL, Stevens J, Dawson D, III, Kryscio RJ, Lin Y, Thomas MV, Miller CS (2013) Patterns of salivary analytes provide diagnostic capacity for distinguishing chronic adult periodontitis from health. J Clin Immunol 33: 271-279. 10.1007/s10875-012-9771-3 [doi].

17. Rathnayake N, Akerman S, Klinge B, Lundegren N, Jansson H, Tryselius Y, Sorsa T, Gustafsson A (2013) Salivary biomarkers of oral health: a cross-sectional study. J Clin Periodontol 40: 140-147. 10.1111/jcpe.12038 [doi].

18. Ebersole JL, Nagarajan R, Akers D, Miller CS (2015) Targeted salivary biomarkers for discrimination of periodontal health and disease(s). Front Cell Infect Microbiol 5: 62. 10.3389/fcimb.2015.00062 [doi].

19. Spielman N, Ilsley D, Gu J, Lea K, Brockman J, Heater S, Setterquist R, Wong D (2012) The Human Salivary RNA Transcriptome Revealed by Massively Parallel Sequencing. Clin Chem . clinchem.2011.176941 [pii];10.1373/clinchem.2011.176941 [doi].

20. Do T, Sheehy EC, Mulli T, Hughes F, Beighton D (2015) Transcriptomic analysis of three Veillonella spp. present in carious dentine and in the saliva of caries-free individuals. Front Cell Infect Microbiol 5: 25. 10.3389/fcimb.2015.00025 [doi].

21. Rudney JD, Xie H, Rhodus NL, Ondrey FG, Griffin TJ (2010) A metaproteomic analysis of the human salivary microbiota by three-dimensional peptide fractionation and tandem mass spectrometry. Mol Oral Microbiol 25: 38-49. OMI558 [pii];10.1111/j.20411014.2009.00558.x [doi].

22. Jagtap P, McGowan T, Bandhakavi S, Tu ZJ, Seymour S, Griffin TJ, Rudney JD (2012) Deep metaproteomic analysis of human salivary supernatant. Proteomics 12: 992-1001. 10.1002/pmic.201100503 [doi].

23. GrassI N, Kulak NA, Pichler G, Geyer PE, Jung J, Schubert S, Sinitcyn P, Cox J, Mann M (2016) Ultradeep and quantitative saliva proteome reveals dynamics of the oral microbiome. Genome Med 8: 44. 10.1186/s13073-016-0293-0 [doi];10.1186/s13073-016-0293-0 [pii].

24. Kongstad J, Ekstrand K, Qvist V, Christensen LB, Cortsen B, Gronbaek M, Holm-Pedersen P, Holmstrup P, Bardow A, Twetman S, Fiehn NE (2013) Findings from the oral health study 
400

401

402

403

404

405

406

407

408

409

410

411

412

413

414

415

416

417

418

419

420

421

422

423

424

425

of the Danish Health Examination Survey 2007-2008. Acta Odontol Scand . 10.3109/00016357.2013.776701 [doi].

25. Jersie-Christensen RR, Sultan A, Olsen JV (2016) Simple and Reproducible Sample Preparation for Single-Shot Phosphoproteomics with High Sensitivity. Methods Mol Biol 1355: 251-260. 10.1007/978-1-4939-3049-4_17 [doi].

26. Cox J, Mann M (2008) MaxQuant enables high peptide identification rates, individualized p.p.b.range mass accuracies and proteome-wide protein quantification. Nat Biotechnol 26: 1367-1372. nbt.1511 [pii];10.1038/nbt.1511 [doi].

27. Jagtap P, Goslinga J, Kooren JA, McGowan T, Wroblewski MS, Seymour SL, Griffin TJ (2013) A twostep database search method improves sensitivity in peptide sequence matches for metaproteomics and proteogenomics studies. Proteomics 13: 1352-1357. 10.1002/pmic.201200352 [doi].

28. 2015) UniProt: a hub for protein information. Nucleic Acids Res 43: D204-D212. gku989 [pii];10.1093/nar/gku989 [doi].

29. Chen T, Yu WH, Izard J, Baranova OV, Lakshmanan A, Dewhirst FE (2010) The Human Oral Microbiome Database: a web accessible resource for investigating oral microbe taxonomic and genomic information. Database (Oxford) 2010: baq013. baq013 [pii];10.1093/database/baq013 [doi].

30. Vizcaino JA, Csordas A, del-Toro N, Dianes JA, Griss J, Lavidas I, Mayer G, Perez-Riverol Y, Reisinger F, Ternent T, Xu QW, Wang R, Hermjakob H (2016) 2016 update of the PRIDE database and its related tools. Nucleic Acids Res 44: D447-D456. gkv1145 [pii];10.1093/nar/gkv1145 [doi].

31. Huerta-Cepas J, Szklarczyk D, Forslund K, Cook H, Heller D, Walter MC, Rattei T, Mende DR, Sunagawa S, Kuhn M, Jensen L, von MC, Bork P (2016) eggNOG 4.5: a hierarchical orthology framework with improved functional annotations for eukaryotic, prokaryotic and viral sequences. Nucleic Acids Res 44: D286-D293. gkv1248 [pii];10.1093/nar/gkv1248 [doi].

32. Eddy SR (2009) A new generation of homology search tools based on probabilistic inference. Genome Inform 23: 205-211. 9781848165632 _0019 [pii].

33. Scholz C, Lyon D, Refsgaard JC, Jensen L, Choudhary C, Weinert BT (2015) Avoiding abundance bias in the functional annotation of post-translationally modified proteins. Nat Methods 12: 1003-1004. nmeth.3621 [pii];10.1038/nmeth.3621 [doi].

34. Lazarevic V, Whiteson K, Gaia N, Gizard Y, Hernandez D, Farinelli L, Osteras M, Francois P, Schrenzel J (2012) Analysis of the salivary microbiome using culture-independent techniques. J Clin Bioinforma 2: 4. 2043-9113-2-4 [pii];10.1186/2043-9113-2-4 [doi].

35. Belda-Ferre P, Williamson J, Simon-Soro A, Artacho A, Jensen ON, Mira A (2015) The human oral metaproteome reveals potential biomarkers for caries disease. Proteomics 15: 3497-3507. 10.1002/pmic.201400600 [doi]. 
426

427

428

429

430

431

432

433

434

435

436

437

438

439

440

441

442

443

444

445

446

447

448

449

450

451

452

453

454

455

456

457

458

459

460

461

36. Takahashi N, Nyvad B (2011) The role of bacteria in the caries process: ecological perspectives. J Dent Res 90: 294-303. 0022034510379602 [pii];10.1177/0022034510379602 [doi].

37. Colombo AP, Boches SK, Cotton SL, Goodson JM, Kent R, Haffajee AD, Socransky SS, Hasturk H, Van Dyke TE, Dewhirst F, Paster BJ (2009) Comparisons of subgingival microbial profiles of refractory periodontitis, severe periodontitis, and periodontal health using the human oral microbe identification microarray. J Periodontol 80: 1421-1432. 10.1902/jop.2009.090185 [doi].

38. Yakob M, Fuentes L, Wang MB, Abemayor E, Wong DT (2014) Salivary biomarkers for detection of oral squamous cell carcinoma - current state and recent advances. Curr Oral Health Rep 1: 133-141. 10.1007/s40496-014-0014-y [doi].

39. Schafer CA, Schafer JJ, Yakob M, Lima P, Camargo P, Wong DT (2014) Saliva diagnostics: utilizing oral fluids to determine health status. Monogr Oral Sci 24: 88-98. 000358791 [pii];10.1159/000358791 [doi].

40. Belstrom D, Holmstrup P, Bardow A, Kokaras A, Fiehn NE, Paster BJ (2016) Comparative analysis of bacterial profiles in unstimulated and stimulated saliva samples. J Oral Microbiol 8: 30112. 30112 [pii].

41. Cole MF, Hsu SD, Baum BJ, Bowen WH, Sierra LI, Aquirre M, Gillespie G (1981) Specific and nonspecific immune factors in dental plaque fluid and saliva from young and old populations. Infect Immun 31: 998-1002.

42. Aurer A, Aurer-Kozelj J, Stavljenic-Rukavina A, Kalenic S, Ivic-Kardum M, Haban V (1999) Inflammatory mediators in saliva of patients with rapidly progressive periodontitis during war stress induced incidence increase. Coll Antropol 23: 117-124.

43. Schenkein HA, Genco RJ (1977) Gingival fluid and serum in periodontal diseases. I. Quantitative study of immunoglobulins, complement components, and other plasma proteins. J Periodontol 48: 772-777. 10.1902/jop.1977.48.12.772 [doi].

44. Courts FJ, Boackle RJ, Fudenberg HH, Silverman MS (1977) Detection of functional complement components in gingival crevicular fluid from humans with periodontal diseases. J Dent Res 56: 327-331.

45. Patters MR, Niekrash CE, Lang NP (1989) Assessment of complement cleavage in gingival fluid during experimental gingivitis in man. J Clin Periodontol 16: 33-37.

46. Attstrom R, Laurel AB, Lahsson U, Sjoholm A (1975) Complement factors in gingival crevice material from healthy and inflamed gingiva in humans. J Periodontal Res 10: 19-27.

47. Okada H, Silverman MS (1979) Chemotactic activity in periodontal disease. I. The role of complement in monocyte chemotaxis. J Periodontal Res 14: 20-25.

48. Okuda K, Takazoe I (1980) Activation of complement by dental plaque. J Periodontal Res 15: 232239. 
462

463

464

465

466

467

468

469

470

471

472

473
49. Watanabe K, Blew B, Scherer M, Burke J, Koh G, Block C, Ramakrishnan V, Frommel TO (1997) CD11b mRNA expression in neutrophils isolated from peripheral blood and gingival crevicular fluid. J Clin Periodontol 24: 814-822.

50. Henry CA, Ungchusri T, Charbeneau TD, Winford TE (1987) Relationships of serum opsonins and complement in human experimental gingivitis. J Periodontol 58: 177-186. 10.1902/jop.1987.58.3.177 [doi].

51. Damgaard C, Holmstrup P, Van Dyke TE, Nielsen CH (2015) The complement system and its role in the pathogenesis of periodontitis: current concepts. J Periodontal Res 50: 283-293. 10.1111/jre.12209 [doi]. 


\section{Figure 1 (on next page)}

\section{Figure 1}

Protein biomass and abundance across sample groups: Relative distribution as a measure of summed intensity and protein count. 
PeerJ

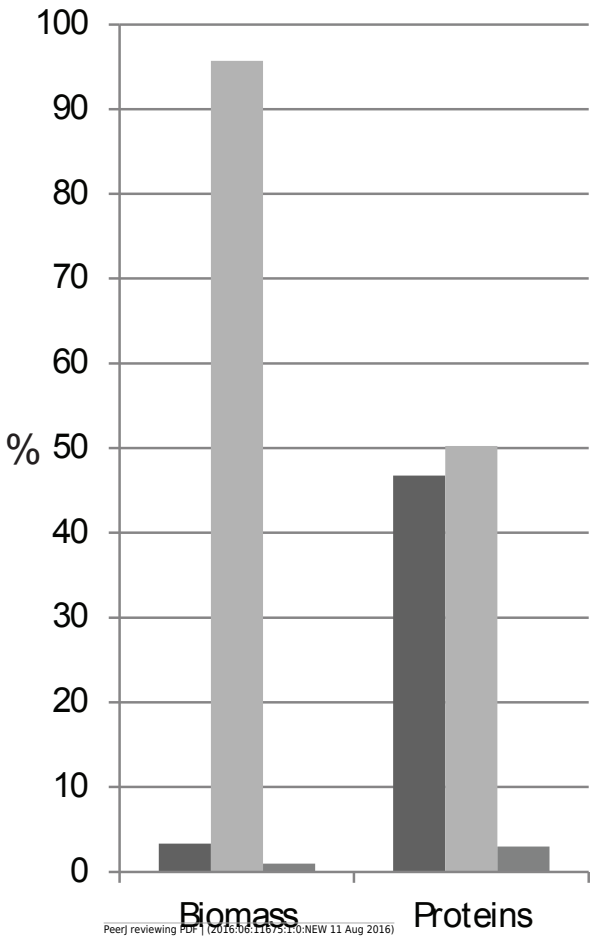

- Bacteria

Homo

- Other 
Figure 2 (on next page)

Figure 2

Principal Component Analysis: A) PCA plot of individuals with caries (blue), periodontitis (red) and orally healthy individuals (green). B) Loadings driving the separation of the PCA plot are mainly proteins belonging to complement and coagulation cascades (purple) for discriminating diseased from healthy individuals. Proteins belonging to salivary secretion and protein glycosylation (green) are mainly defining the healthy individuals. 
A

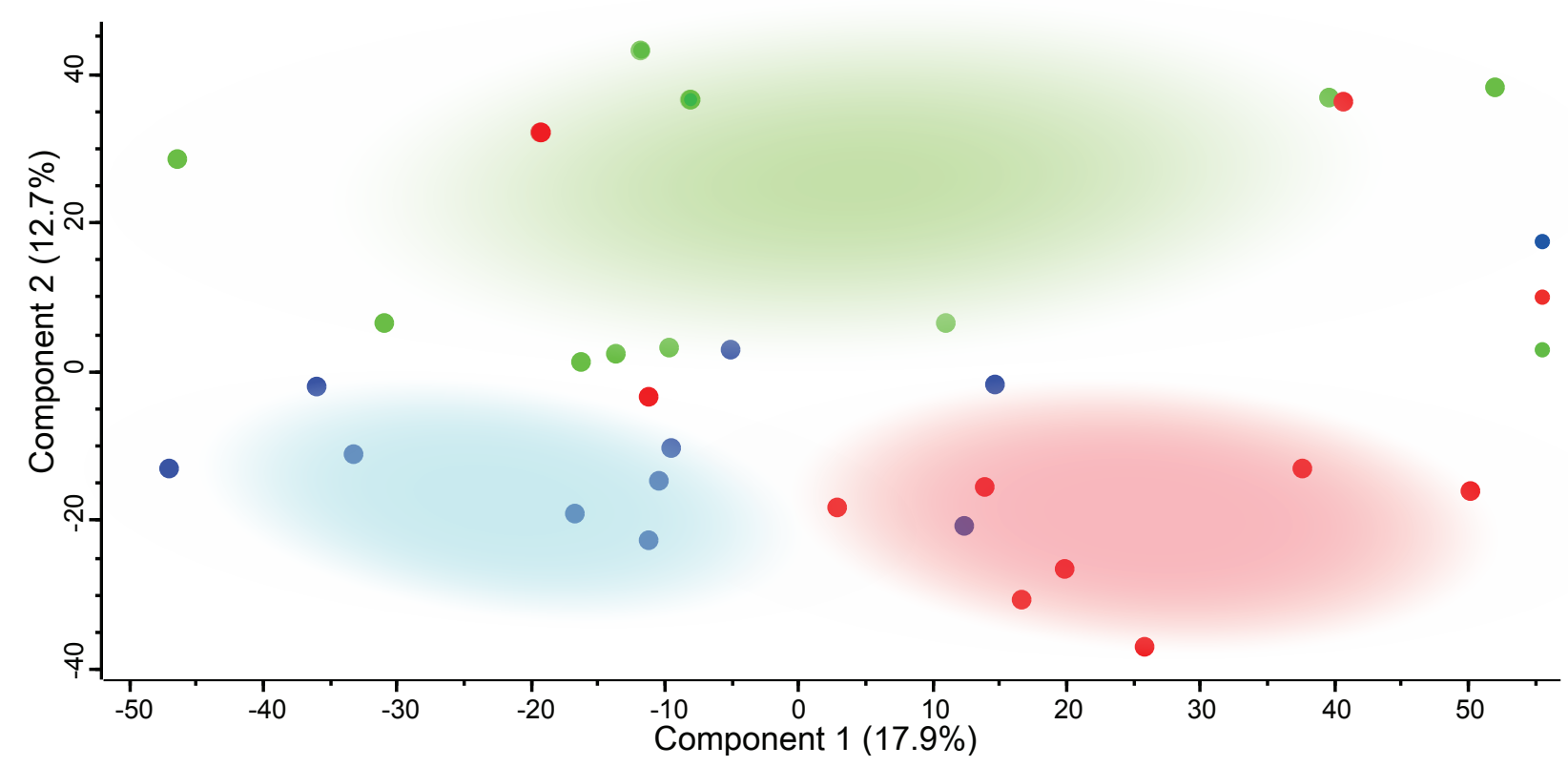

Caries

- Periodontitis

- Healthy

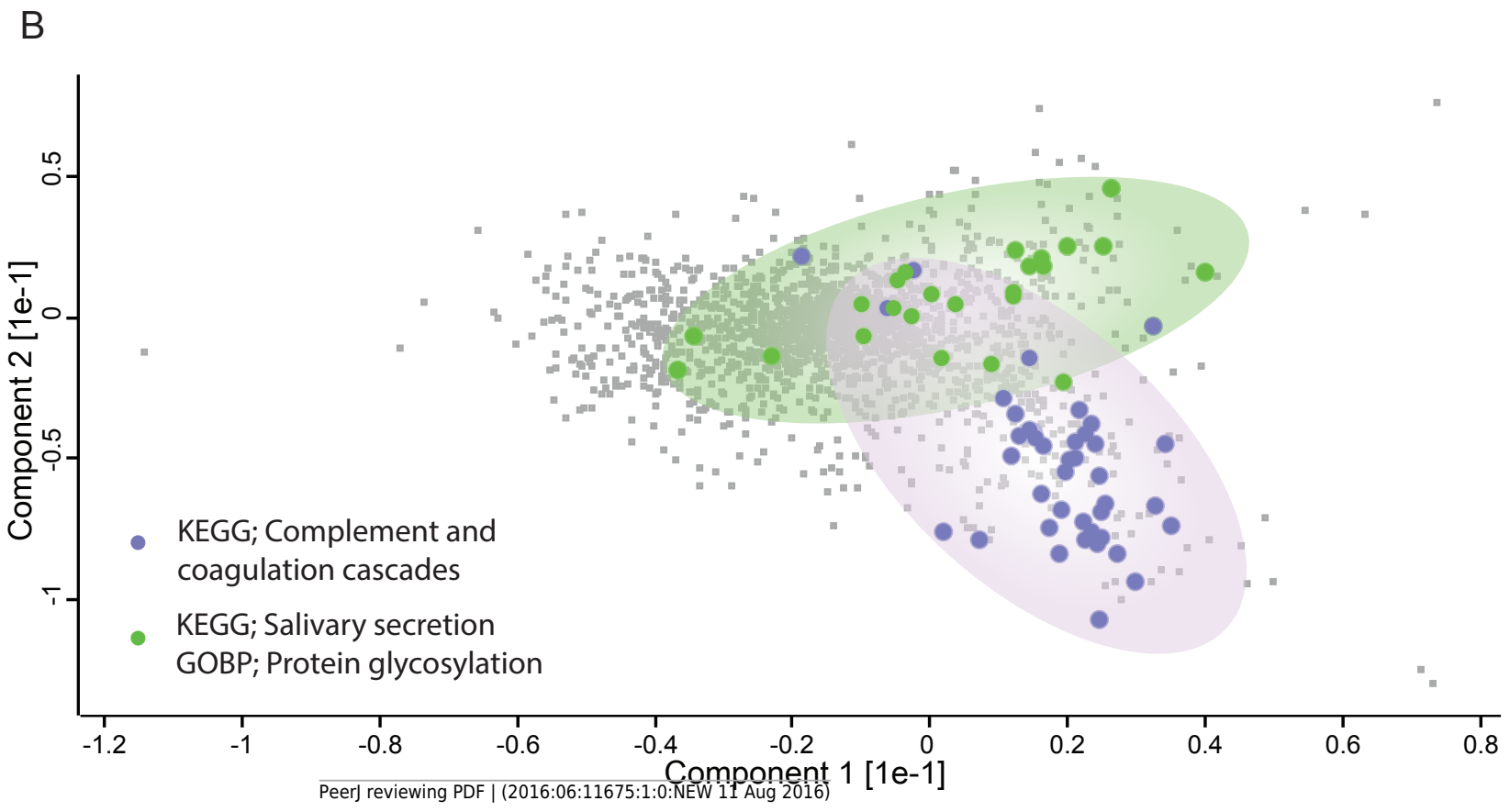




\section{Figure 3 (on next page)}

Figure 3

Potential biomarkers of oral health and disease: Intensity-based heat-map of proteins significantly differentially expressed between the three groups. Protein names in purple are associated with innate immune response, protein names in orange are associated with lipid transfer. 

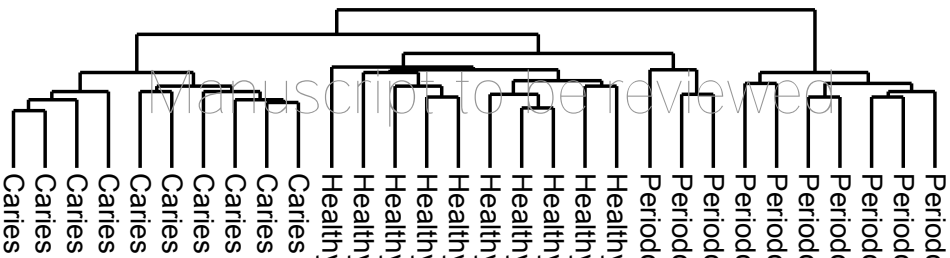

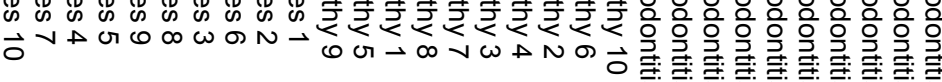

ब जN $\overrightarrow{0} \omega \vee \perp \omega \infty \rightarrow$

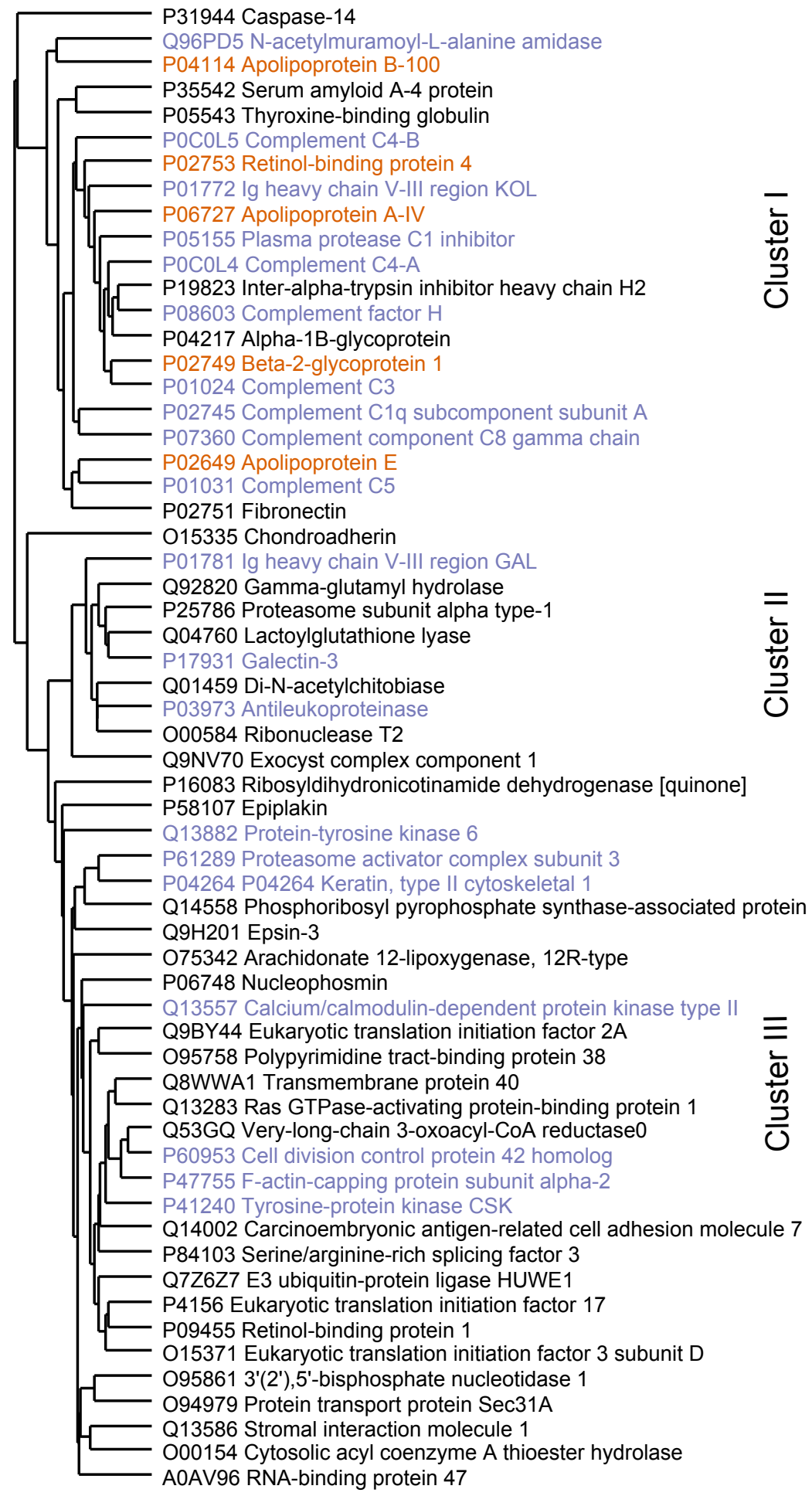

P31944 Caspase-14

P04114 Apolipoprotein B-100

P35542 Serum amyloid A-4 protein

Thyroxine-binding globulin

ement C4-B

P01772 lg heavy chain V-II region KOL

P06727 Apolipoprotein A-IV

155 Plasma protease C1 inhibitor

P19823 Inter-alpha-trypsin inhibitor heavy chain $\mathrm{H} 2$

P08603 Complement factor

P02745 Complement C1q subcomponent subunit A

Pomplement component C8 gamma chain

02751 Fibronectin

Q04760 Lactoylglutathione lyase

000584 R

Q9NV70 Exocyst complex component

P58107 Epiplakin

P06748 Nucleophosmin

Q13557 Calcium/calmodulin-dependent protein kinase type Fukaryotic translation initiation factor $2 A$

Q8WWA1 Transmembrane protein 40

Q13283 Ras GTPase-activating protein-binding protein 1

kinase CSK

Carcinoembryonic antigen-relate

Eukaryotic translation initiation factor 17

A0AV96 RNA-binding protein 47

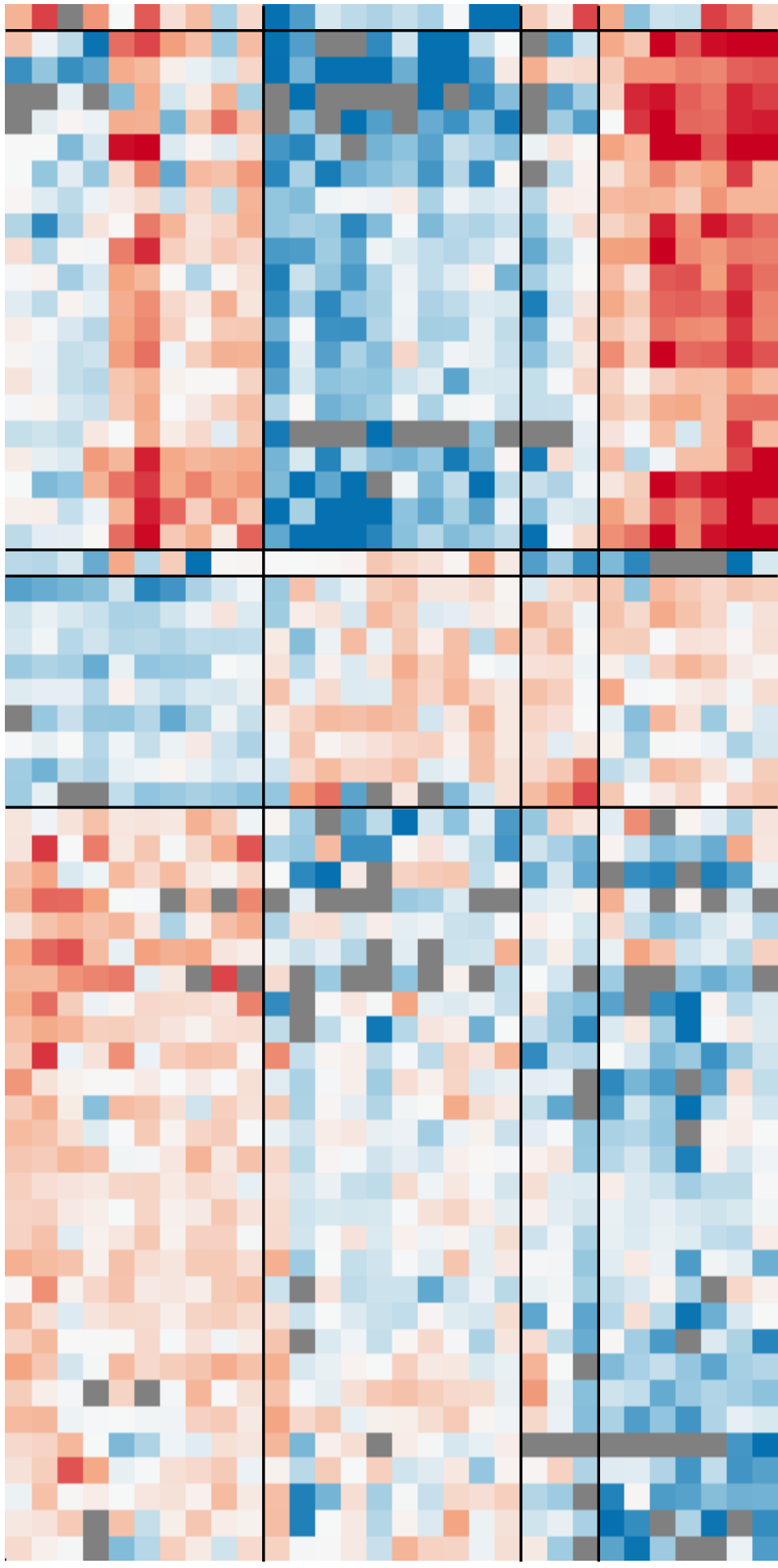

$-3$

Fold change from mean

(Log2 intensity - mean) 


\section{Figure 4(on next page)}

Figure 4

Predominant bacterial genera and species: Relative protein intensity abundance of top 20 genera A) and species B). 
A

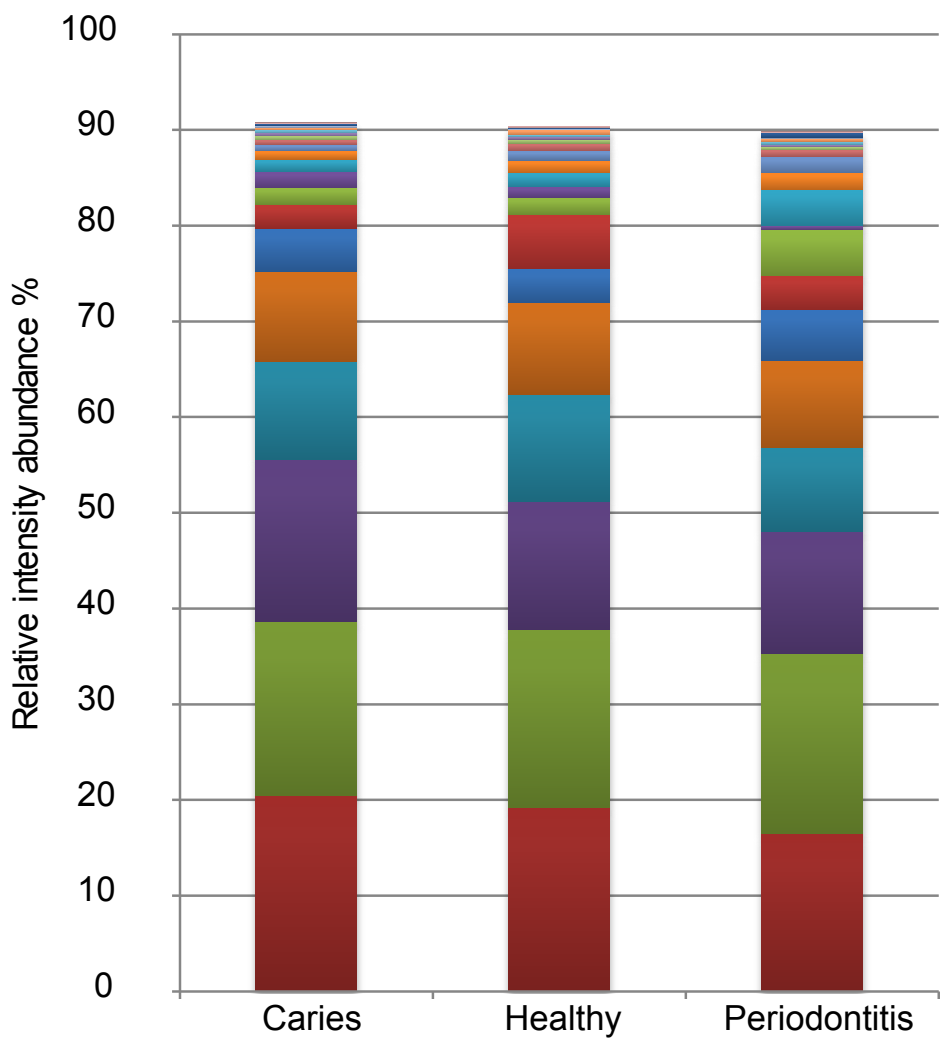

- Granulicatella

- Corynebacterium

- Megasphaera

Alloprevotella

Stomatobaculum

- Oribacterium

- Mycoplasma

- Gemella

- Selenomonas

- Porphyromonas

- Fusobacterium

- Lactobacillus

- Leptotrichia

- Haemophilus

- Actinomyces

- Neisseria

- Rothia

- Veillonella

Prevotella

- Streptococcus

B

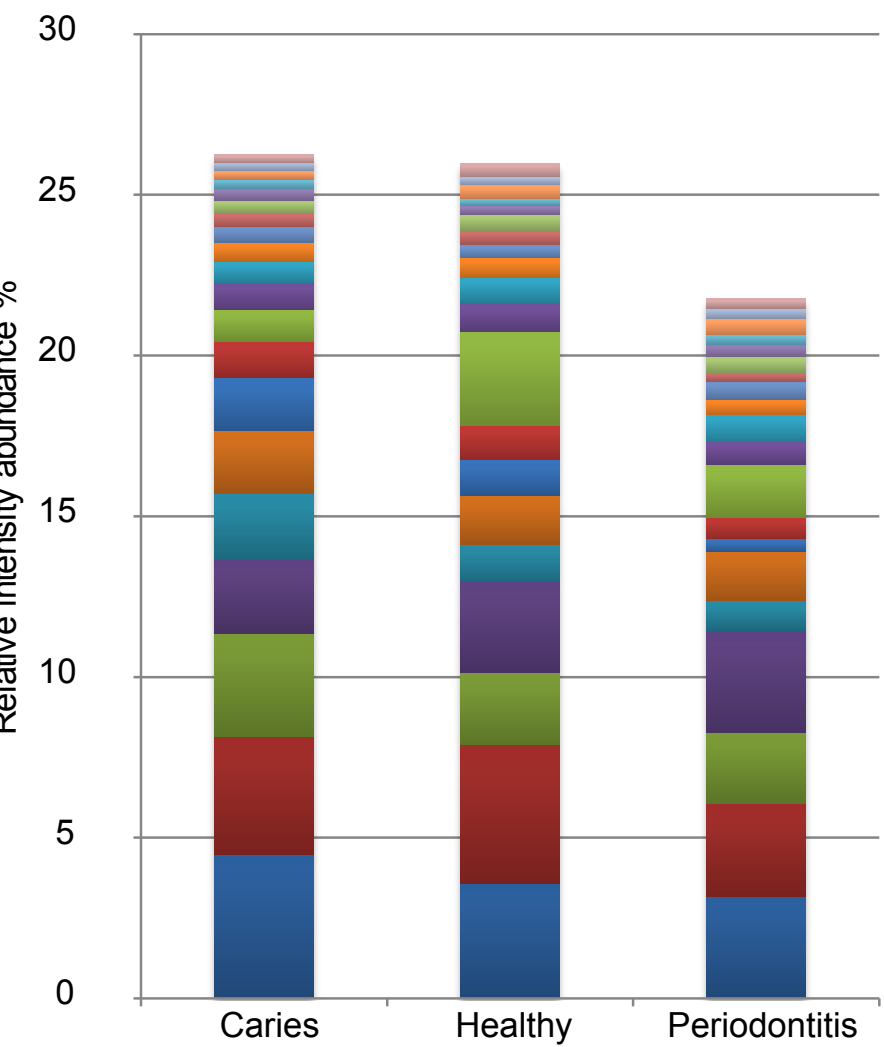

- Prevotella sp. oral taxon 473

- Lachnospiraceae bacterium oral taxon 82

- Porphyromonas sp. oral taxon 279

- Stomatobaculum longum

Actinomyces sp. oral taxon 172

- Neisseria mucosa

- Mycoplasma salivarium

- Actinomyces graevenitzii

Neisseria subflava

- Veillonella sp. oral taxon 158

- Prevotella scopos

- Haemophilus parainfluenzae

- Streptococcus parasanguinis

- Lactobacillus salivarius

- Veillonella dispar

- Streptococcus salivarius

- Prevotella melaninogenica

- Veillonella atypica

- Rothia mucilaginosa

- Prevotella histicola 


\section{Table $\mathbf{1}$ (on next page)}

Table 1

Overview of proteins identified 


\begin{tabular}{|l|c|c|c|c|}
\hline \multicolumn{1}{|c|}{ Number of Proteins } & Caries & Healthy & Periodontitis & Total* \\
\hline Other** & 125 & 115 & 120 & 125 \\
\hline Human & 2084 & 2079 & 2084 & 2090 \\
\hline Bacteria & 1861 & 1926 & 1924 & 1946 \\
\hline -mapped to genus level & 1710 & 1765 & 1762 & 1784 \\
& $(91.9 \%)$ & $(91.6 \%)$ & $(91.6 \%)$ & $(91.7 \%)$ \\
\hline -mapped to species level & 594 & 602 & 609 & 616 \\
& $(31.9 \%)$ & $(34.1 \%)$ & $(34.6 \%)$ & $(34.5 \%)$ \\
\hline Total & 4070 & 4120 & 4128 & 4161 \\
\hline
\end{tabular}

$1 *$ Unique proteins.

$2 * *$ Food related proteins and proteins that could not be assigned to kingdom level. 